\title{
Effect of 3D-scaffold formation on differentiation and survival in human neural progenitor cells
}

\author{
Stefanie Ortinau ${ }^{1 \dagger}$, Jürgen Schmich ${ }^{1 \dagger}$, Stephan Block ${ }^{2}$, Andrea Liedmann ${ }^{1}$, Ludwig Jonas ${ }^{3}$, Dieter G Weiss ${ }^{4}$, \\ Christiane A Helm², Arndt Rolfs', Moritz J Frech
}

* Correspondence: moritz. frech@uni-rostock.de

\begin{abstract}
Background: 3D-scaffolds have been shown to direct cell growth and differentiation in many different cell types, with the formation and functionalisation of the 3Dmicroenvironment being important in determining the fate of the embedded cells. Here we used a hydrogel-based scaffold to investigate the influences of matrix concentration and functionalisation with laminin on the formation of the scaffolds, and the effect of these scaffolds on human neural progenitor cells cultured within them.

Methods: In this study we used different concentrations of the hydrogel-based matrix PuraMatrix. In some experiments we functionalised the matrix with laminin I. The impact of concentration and treatment with laminin on the formation of the scaffold was examined with atomic force microscopy. Cells from a human fetal neural progenitor cell line were cultured in the different matrices, as well as in a 2D culture system, and were subsequently analysed with antibody stainings against neuronal markers. In parallel, the survival rate of the cells was determined by a live/ dead assay.

Results: Atomic force microscopy measurements demonstrated that the matrices are formed by networks of isolated PuraMatrix fibres and aggregates of fibres. An increase of the hydrogel concentration led to a decrease in the mesh size of the scaffolds and functionalisation with laminin promoted aggregation of the fibres (bundle formation), which further reduces the density of isolated fibres. We showed that laminin-functionalisation is essential for human neural progenitor cells to build up 3D-growth patterns, and that proliferation of the cells is also affected by the concentration of matrix. In addition we found that 3D-cultures enhanced neuronal differentiation and the survival rate of the cells compared to 2D-cultures.

Conclusions: Taken together, we have demonstrated a direct influence of the 3Dscaffold formation on the survival and neuronal differentiation of human neural progenitor cells. These findings emphasize the importance of optimizing 3D-scaffolds protocols prior to in vivo engraftment of stem and progenitor cells in the context of regenerative medicine.
\end{abstract}




\section{Background}

Tissue engineering is an interdisciplinary field combining biological sciences and engineering to develop tissues that restore, maintain or enhance tissue function. In the context of regenerative medicine, the combination of biomaterial scaffolds with neural stem and progenitor cells holds great promise as a therapeutic tool [1,2]. 3D-matrices have been generated from various materials such as poly L-lactic acid and poly glycolic acid [3,4], as well as biopolymers such as collagen, fibrin, and alginate [5-14]. The hydrogel-based PuraMatrix is an ionic self-complementary amphiphilic oligopeptide hydrogel matrix, able to form 3D nano-scaffolds consisting of $\beta$-sheets and fibres by spontaneous molecular self-assembling [12-14]. The hydrogel scaffold is widely used in the areas of tissue engineering and stem cell research, and it has been shown to promote differentiation in different cell types [15-24]. As the composition, concentration and functionalisation of the 3D-scaffolds is essential for cell adhesion, growth and differentiation, we varied the concentration and functionalisation of PuraMatrix seeded with a human neural progenitor cell line (ReNcell VM, Millipore, USA) and studied the effects on the assembly of the matrix, and subsequently the influence on the differentiation of the human progenitor cells. This cell line shows fast proliferation and can be cultured easily, which makes it an appropriate system to test the influence of a 3D environment. The cells can be differentiated into neurons, astrocytes and oligodendrocytes within a few days by simple withdrawal of growth factors [25]. Although the overall differentiation into neuronal cells is relatively low, the above mentioned properties made the cell line an appropriate model to study differentiation of human progenitor cells, and has already been used in other studies [26-29]. Matrix assembly was analysed by performing atomic force microscopy to provide structural information about the matrix, such as spatial dimensions of the fibres and the structure of the network formed by these fibres. In addition we report data on the influence of the scaffold formation and functionalisation on proliferation, growth and differentiation of human neural progenitor cells cultured in the 3D-scaffolds in comparison to the situation in 2D cultures. The data presented provides new information for optimizing 3D-scaffolds to be used in die field of regenerative medicine.

\section{Methods}

\section{Atomic force microscope (AFM) measurements}

PuraMatrix (PM; BD Biosciences, Heidelberg, Germany) and laminin stock solutions were kept at $4{ }^{\circ} \mathrm{C}$ until used. Solutions for the deposition process were freshly prepared from the stock solutions as described below, but without cells. Muscovite mica sheets were freshly cleaved and immediately placed into a 24 well-plate. In each case $100 \mu \mathrm{l}$ of the solutions were placed on top of the mica sheet, then $400 \mu \mathrm{l}$ of media without growth factors was added. One day later the mica sheets were rinsed in ultra pure water (Millipore, Billerica, MA) to remove excess gel loosely bound to the mica, and then dried under a stream of nitrogen.

Imaging was performed using a Multimode Atomic Force Microscope (AFM) with Nanoscope IIIa controller and "E" scanner (Digital Instruments, Santa Barbara, CA). The "E" scanner exhibits a maximum scan area of $10 \times 10 \mu \mathrm{m}^{2}$, a vertical range of 2.5 $\mu \mathrm{m}$ and was height calibrated using a TGZ01 grating (MicroMasch, Estonia; step height $26 \mathrm{~nm}$ ) and linearised using a PG grating (Digital Instruments; $1 \mu \mathrm{m}$ pitch). 
The images were recorded with tapping mode in air using standard tapping mode cantilevers (OMCL-AC160TS, Olympus). Imaging in fluid was not necessary because the drying process does not affect the structure of the scaffold [30].

Before usage the cantilevers were tested with a Nioprobe self-imaging sample (Aurora Nanodevices, Canada) and with a gold cluster sample (cluster radius $<15 \mathrm{~nm}$; synthesised according to [31]) and only cantilevers with tip radius $R<10 \mathrm{~nm}$ were used for imaging. Images were obtained from at least five different positions and in three different resolutions $(500 \mathrm{~nm} \times 500 \mathrm{~nm}, 1500 \mathrm{~nm} \times 1500 \mathrm{~nm}, 10 \mu \mathrm{m} \times 10 \mu \mathrm{m})$.

Image processing was performed using homebuilt scripts in MatLab (MathWorks Natick, MA). The large area scans $(1500 \mathrm{~nm} \times 1500 \mathrm{~nm}$ and $10 \mu \mathrm{m} \times 10 \mu \mathrm{m})$ were used to get an impression of the coarse network structure (i.e. number of crossing points, aggregation of single fibres etc.) whereas the small area scans $(500 \mathrm{~nm} \times 500$ $\mathrm{nm}$ ) were used to measure the geometric properties of PM fibres in presence and absence of laminin.

It is well known that PM fibres form beta-sheets [32] and hence the interaction of the AFM tip with one PM fibre can be well approximated by the interaction of a sphere (radius $R$ ) with a cuboid (width $w$ and height $h$ ). From Figure 1A one can conclude that the spatial extension of the AFM tip leads to a broadening of the slab width $w_{\mathrm{AFM}}$ in the AFM image whereas the cuboid height $h_{\mathrm{AFM}}$ remains unaffected. Using simple geometric calculations one can estimate that the difference between measured and true width is given by (Figure $1 \mathrm{~A}$ bottom).

$$
w_{\mathrm{AFM}}-w=2\left(2 h R-h^{2}\right)^{1 / 2} .
$$

This introduces a noticeable error in our case as the dimensions of tip radius (approx. $5 \mathrm{~nm}$ ) and PM fibre height (approx. $1.3 \mathrm{~nm}$ ) and width (approx. $5 \mathrm{~nm}$ ) are of the same order of magnitude. To account for this effect the curvature radius of the

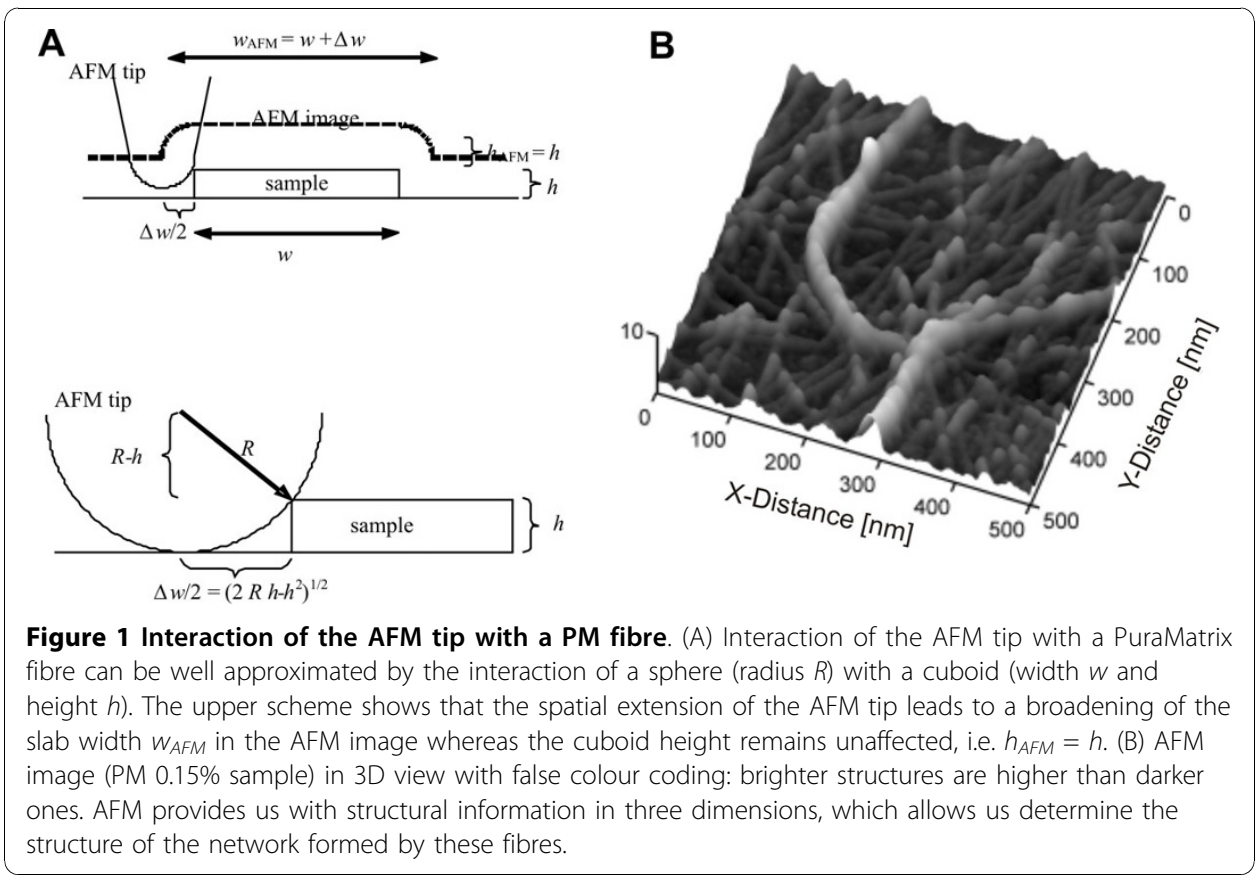


AFM tip $R$ was obtained from tapping mode images of the Nioprobe self-imaging sample. Furthermore, the width $w$ and the height $h$ of isolated PM fibres were measured from $500 \mathrm{~nm} \times 500 \mathrm{~nm}$ scans of PM samples (PM concentration $0.15 \%$, with and without laminin) and corrected according to Eq. 1 . The error given in the results was calculated using the standard deviation (tip radius $R$ and beta-sheet height $h$ ) or by employing the error propagation law (beta-sheet width $w$ ).

\section{Cell culture}

2D culture of ReNcell VM (Millipore, Schwalbach, Germany) cells was carried out as described previously [27]. Cells were cultivated on laminin I (mouse laminin I, AMS Biotechnology, Germany) coated flasks or chamber slides in Dulbecco's modified eagle medium (DMEM)/F12, supplemented with Glutamax, B27 media supplement, heparin sodium salt and gentamycin (all Invitrogen, Karlsruhe, Germany). Epidermal growth factor (20 ng/ml; EGF) and basic fibroblast growth factor $(10 \mathrm{ng} / \mathrm{ml}$; bFGF; both Roche, Mannheim, Germany) were added to the media during proliferation. Differentiation of the cells was induced by withdrawal of the EGF and bFGF.

To prepare 3D PuraMatrix hydrogel matrices (BD Biosciences, Heidelberg, Germany) 2D cultured cells were trypsinized and resuspended in $10 \%$ sucrose $(60,000$ cells $/ 100 \mu \mathrm{l}$ matrix) and incubated for $30 \mathrm{~min}$. For functionalisation of the PuraMatrix, cells were mixed with laminin I solution (mouse laminin I, AMS Biotechnology, Germany) in advance to the incubation $(8 \mu \mathrm{g} / 100 \mu \mathrm{l}$ matrix). Subsequently cells were transferred to coverslips in a 24 -well plate and media $(400 \mu \mathrm{l} /$ well) was added. Afterwards the matrices were allowed to gelate for $1 \mathrm{~h}$. Matrices were washed with $500 \mu \mathrm{l}$ media for $10 \mathrm{~min}$ at room temperature. After an additional washing step the matrices were incubated at $37^{\circ} \mathrm{C} / 5 \% \mathrm{CO}_{2}$. Unless otherwise stated all reagents were purchased from Sigma (Taufkirchen, Germany).

\section{Immunocytochemistry and scanning electron microscopy}

For immunocytochemistry, 2D- and 3D-cultures were fixed with paraformaldehyde (4\% in $0.1 \mathrm{M} \mathrm{PBS}$ ) for $20 \mathrm{~min}$. Cells were incubated with the primary antibody over night at $4{ }^{\circ} \mathrm{C}$ (anti- $\beta$-III-tubulin, 1:1000, mouse, Sigma, Munich, Germany) or anti-tyrosine hydroxylase (TH, 1:500, mouse, R \& D Systems, Wiesbaden, Germany). Secondary antibody (1:1000; goat, anti-mouse Alexa Fluor 488, Molecular Probes) was added for $5 \mathrm{~h}$ at room temperature. Cell nuclei labelling was performed with 4',6-Diamidin-2'phenylindoldihydrochlorid (DAPI, $100 \mathrm{ng} / \mathrm{ml}$ in PBS, Sigma, Munich, Germany). 3Dmatrices and 2D-cultures were analysed either by fluorescent microscopy (Olympus BX51, Olympus Germany or a Biozero microscope, Keyence, Germany, Karlsruhe), or confocal microscopy (Leica, DM IRE2 equipped with UV, Argon and Xenon lasers).

In 2D-cultures, pictures were taken of 8-10 non overlapping visual areas per chamber, with three chambers per time point. DAPI stained nuclei were counted using GSA Image Analyzer program (GSA, Rostock, Germany), whereas immuno-positive cells were counted manually.

For 3D-matrix cultures, at least three stacks of images (20-40 images/stack, distance $2 \mu \mathrm{m})$ per time point and per concentration of PM sample were taken by confocal microscopy. To obtain the total cell numbers, stacks were merged with the NIS element D program (Nikon, Düsseldorf, Germany) and then analysed with GSA Image 
Analyzer program. Given values represent mean percentage \pm SEM with respect to DAPI stained cells. Data were obtained from at least 3 independent experiments $(\mathrm{N})$ and $\geq 6$ measurements $(\mathrm{n})$ per $\mathrm{N}$ for $2 \mathrm{D}$ cultures and $\geq 3$ (n) for 3D-experiments.

For scanning electron microscopy, cells contained in PuraMatrix were fixed with glutaraldehyde (4\% in PBS) for $1 \mathrm{~h}$ or overnight, rinsed with PBS and subsequently dehydrated in acetone with increasing concentrations (30\%, 50\%, 75\%, 90\%, 100\%). Specimens were dried with a critical point drier (BalTec, Germany) and sputter coated with gold. Pictures were taken with a DSM 960A scanning electron microscope (ZEISS, Germany).

\section{Live/dead assay}

Viability of cells was measured by live/dead assay (Molecular Probes, Karlsruhe, Germany) and analysed by fluorescence microscopy (TS100, Nikon, Düsseldorf, Germany). Quantitative data of 2D-cultures were obtained by manually counting of 8-10 nonoverlapping areas per three chambers per time point. 3D-matrices were analysed by taking at least three image stacks per matrix concentration and time point. Viability is given as mean \pm SEM of living cells in the total number of cells.

\section{Statistics}

All statistical analyses described were performed with Prism 5 (GraphPad Prism. Inc., USA) using one way ANOVA analysis with the Bonferroni Post test. p-value $\leq 0.05$ (indicated by $*$ ) was considered to indicate significant statistical differences. Values represent mean \pm sem.

\section{Results}

\section{Atomic force microscopy of PuraMatrix}

The composition, concentration and functionalisation of a 3D-scaffold is essential for cell adhesion, growth and finally differentiation. Therefore we studied matrix assembly by performing atomic force microscopy (AFM) on PuraMatrix samples. AFM images provide structural information in three dimensions which allowed us to measure the spatial dimensions of the fibres as well as the structure of the network formed by these fibres (Figure 1B). For pure PM scaffolds the AFM images are given in Figure 2, whereas Figure 3 shows the AFM results for scaffolds made by a mixture of PM and laminin I. In these figures the surface morphology is given in two different resolutions: the left column shows the scaffold morphology at a surface area of $0.75 \times 0.75 \mu \mathrm{m}^{2}$, which allows the resolution of single, isolated PM fibres, whereas the right column has a surface area of $4 \times 4 \mu \mathrm{m}^{2}$ and is used to study the structure of the scaffold on a much larger scale. For pure PM scaffolds made at low PM concentration (Figure 2A, B; PM 0.15\%) most of the scaffold consists of thin PM fibres (e.g. white arrows in Figure $2 \mathrm{~A}$ ) which are quite homogeneously distributed over the scaffold and mostly isolated. Interestingly, these fibres show an alignment which is parallel to the mica surface. Therefore we conclude that the washing of the PM samples by Milli-Q water leads to a dissection of the PM scaffold and that only the fibres and aggregates of the scaffold, which are adjacent to the mica surface, remain on the sample. Due to this alignment we were able to measure the geometric properties of the pure and isolated PM fibres and found that they have an average height of $1.28 \pm 0.16 \mathrm{~nm}$ and an 

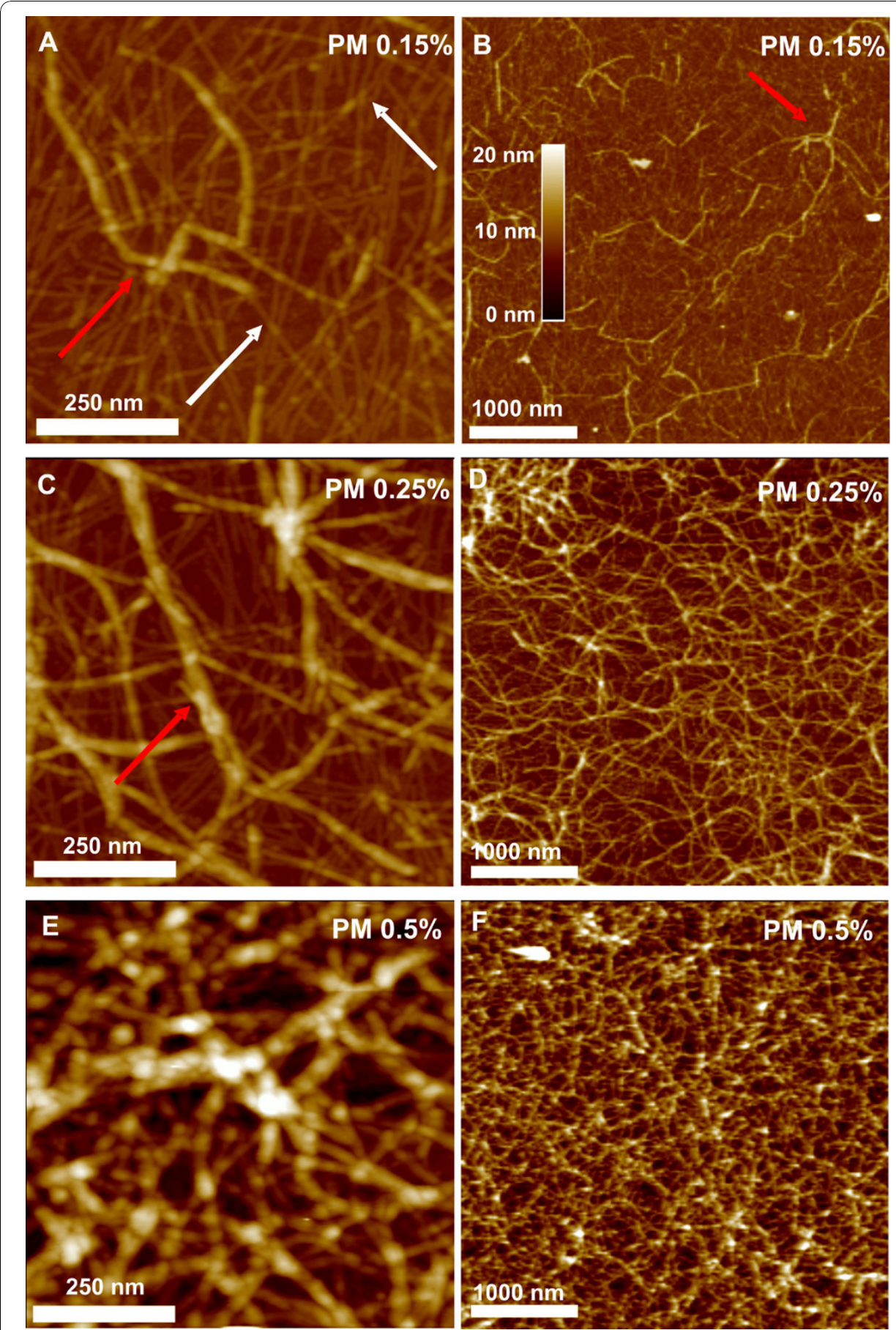

Figure 2 AFM images of pure PuraMatrix scaffolds. AFM images of pure PuraMatrix scaffolds; scan size: $0.75 \times 0.75 \mu \mathrm{m}^{2}$ (left column) or $4 \times 4 \mu \mathrm{m}^{2}$ (right column). Depending on the PM concentration two kinds of fibres are formed: long and thin beta-sheets (i.e. isolated PM fibres, exemplary marked by white arrows; height ca. $1.3 \mathrm{~nm}$ and length $>1 \mu \mathrm{m}$ ) and bundles or aggregates of these sheets (red arrows). Additionally, a crossing of single PM fibres is accompanied by an increase in height (white arrows), indicating that once the PM fibres are created, they do not interdigitate. At low PM concentrations (PM $0.15 \%$ and $0.25 \%$, Fig. $2 A-D$ ) the samples show a homogeneous distribution of single PM fibres and bundles, whereas an increase in PM concentration rises the numbers of bundles within the network. At $0.5 \%$ PM concentration ( $E, F)$ the fibres form very dense networks that cannot be penetrated by AFM. 


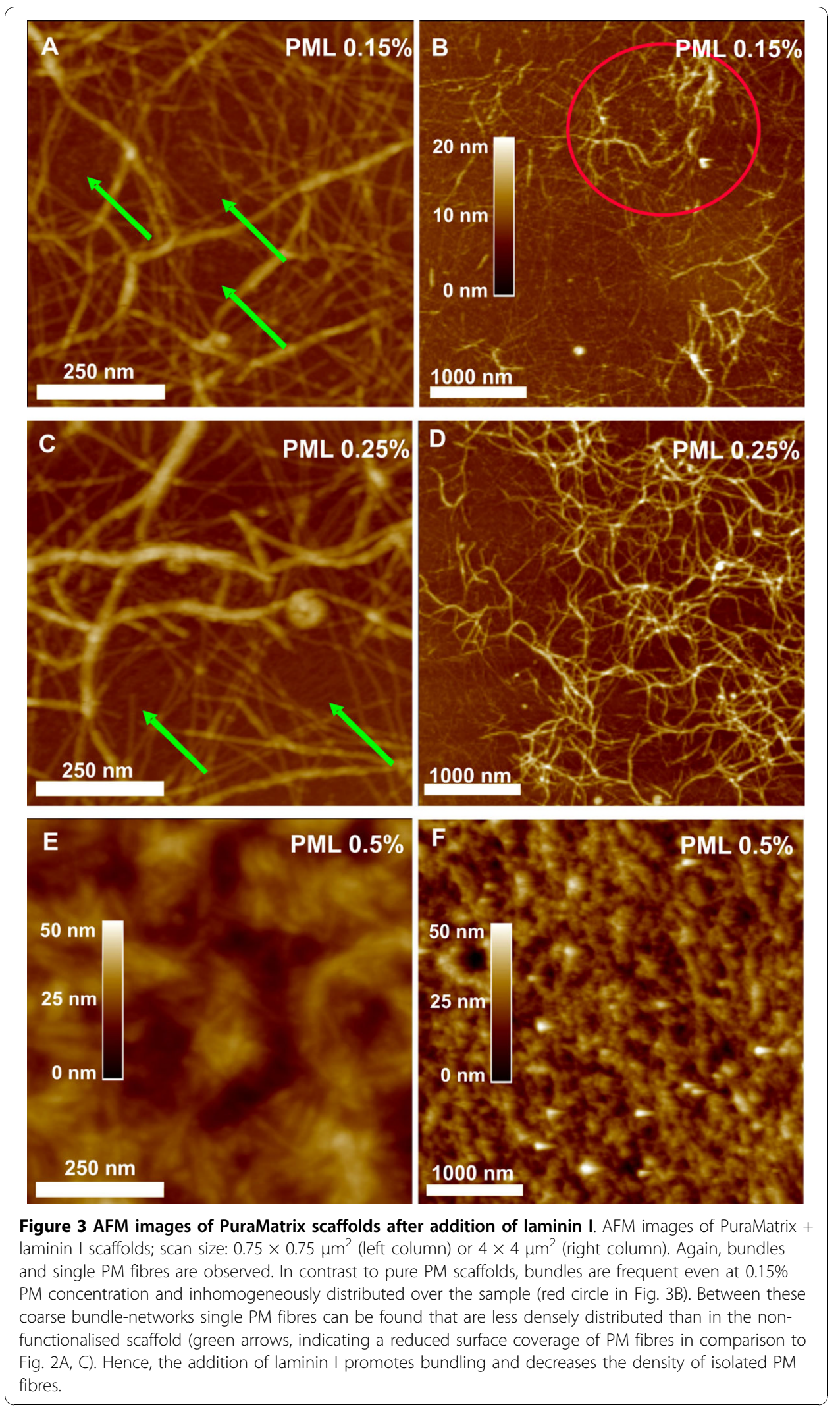


average width of $6.86 \pm 2.69 \mathrm{~nm}$, which agrees well with the molecular dimensions of single PuraMatrix beta-sheets (height: $1.3 \mathrm{~nm}$, width: $5 \mathrm{~nm}$, according to [30]). However, we also found structures in the AFM images whose geometric properties are larger (in height and width) than the isolated PM fibres (red arrows in Figure 2A, B). We attribute these structures to aggregation of single PM fibres into PM bundles. Interestingly, the amount of bundles rises with increasing PM concentration (Figure $2 \mathrm{C}-\mathrm{F}$ ), which causes very dense scaffolds at high PM concentrations (Figure 2E, F). The higher resolved AFM images show (left column of Figure 2) that for pure PM scaffolds there is always a homogeneous distribution of single PM fibres within the scaffold. An increase in PM concentration leads to increased aggregation (bundle formation) of PM fibres. On the other hand, it can be seen from the larger AFM scans (right column of Figure 2), that the PM bundles form a coarser network themselves. The PM bundles of this network are also quite homogeneously distributed over the surface, whereas the mesh size increases with decreasing PM concentration (Figure 2D, F). At 0.15\% PM concentration bundle formation becomes infrequent and we find almost no PM bundle network within the scaffold (Figure 2B). This is changed upon addition of laminin (red circle in Figure 3B). Here the scaffold becomes dense only at $0.5 \%$ PM concentration (Figure 3E, F). At lower concentrations single PM fibres become less frequent and most of the network is formed by PM bundles (Figure 3A-D). Additionally, these bundle networks are inhomogeneously distributed over the surface (Figure 3B, D). Hence, we conclude that the addition of laminin promotes bundle formation and reduces the amount of isolated PM fibres. As the same PM amount is now distributed more in bundles and less in single sheets, we observed areas surrounded by the bundle network which are not covered by any PM fibres (green arrows in Figure 3A, C). This shows that at low PM concentrations the scaffold becomes less dense compared to pure PM scaffolds and hence, that the addition of laminin I has a big impact on the PM scaffold structure.

For a quantification of this effect we calculated height distribution histograms of the AFM images for both types of PM scaffolds at low PM concentrations. Without laminin (PM 0.15\% in Figure 4A) a peak can be found around $1 \mathrm{~nm}$ in the distribution histogram, corresponding to the height of a single PM fibre (height approx. $1.3 \mathrm{~nm}$ ) and two peaks around $2.3 \mathrm{~nm}$ and $3.7 \mathrm{~nm}$, indicating aggregates of two or three PM fibres respectively. The average separation of the peaks is $1.23 \pm 0.21 \mathrm{~nm}$, which is close to the expected value of $1.3 \mathrm{~nm}$ (height of one PM fibre). Figure 4A shows that single PM fibres are more frequent than bundles by roughly a factor of 10. Additionally, Figure 4B compares the height distribution histograms of PM 0.15\% and PML 0.15\%. After addition of laminin the peak at approx. $1 \mathrm{~nm}$ is decreased whereas the other peaks are increased by roughly a factor of 2 indicating more bundles are formed by aggregation of PM fibres. Furthermore, the addition of laminin I increases the substrate peak at $0 \mathrm{~nm}$ in the histograms. We conclude that the additional aggregation in lateral ( $\mathrm{x}, \mathrm{y}$-axis) and vertical dimensions (z-axis) after addition of laminin leads to a lowering of surface coverage by PM, which means that more of the bare substrate is visible to the AFM tip and explains the increased substrate peak. Qualitatively, the same result was found in the AFM images (Figure 2A and 3A), where we observed areas without any PM fibres after the addition of laminin (green arrows in Figure 3A). Comparing Figure 4A with Figure 2A, we conclude that peaks at multiples of $1.3 \mathrm{~nm}$ 

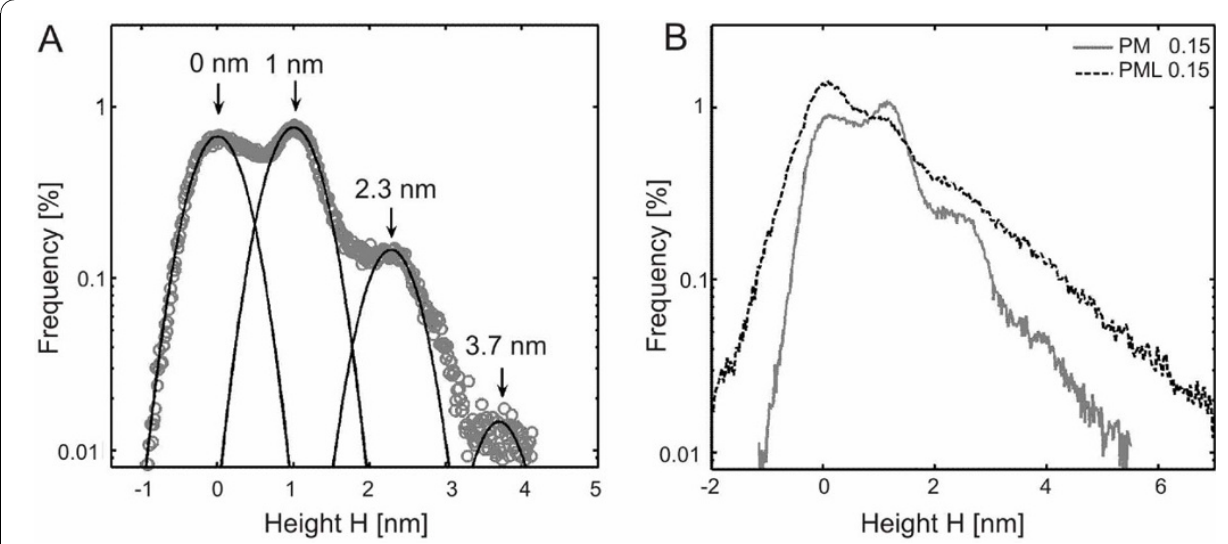

Figure 4 Height distribution histograms. (A) Height distribution histograms (open circles, grey) of a PM $0.15 \%$ sample and fits to Gaussian distribution function (solid lines). The leftmost peak corresponds to the surface bearing the PM network and was set to $0 \mathrm{~nm}$. Three peaks with an average separation of $1.23 \pm$ $0.21 \mathrm{~nm}$ and declining intensities are shown. Therefore mostly single PM fibres are formed and the formation of vertical aggregates is very unlikely. (B) Comparison of height distribution histograms from PM $0.15 \%$ (gray line) and PML $0.15 \%$ (black line). Obviously the formation of single PM fibres is reduced (peak at approx. $1 \mathrm{~nm}$ ) and the aggregation to bundles is preferred if laminin is added. Furthermore the surface peak $(0 \mathrm{~nm})$ is increased, indicating that less surface is covered by PM. This supports the observation (Fig. $3 \mathrm{~A}, \mathrm{C})$, that the bundling makes the network less dense.

are mainly caused by two processes: 1 . sandwich-like aggregation of PM fibres to bundles and 2. crossing of two beta-sheets leading to an elevation at the crossover. The second process can be found in Figure 2A, where the crossing points of different PM fibres are marked by white arrows. Interestingly, at every crossing point the height of the fibres locally increases, which indicates that one fibre has to go above the other one and that the fibres do not interdigitate. Furthermore, the PM fibres have to be surprisingly elastic, as every single fibre crossover has similar spatial dimensions as a betasheet (same in height but slightly increased width). Hence, the beta-sheets strongly deform just at the crossing point.

\section{Cell growth in 3D-matrices}

As we demonstrated the influence of the PM concentration and of laminin on the formation of the 3D-scaffold, in a subsequent set of experiments we investigated the impact of the scaffold formation on cell growth and survival. We used the human fetal neural progenitor ReNcell VM cell line as a model system for neuronal differentiation as the cells can be differentiated in neuronal cells with a dopaminergic phenotype, and the cells start differentiation within $24 \mathrm{~h}[26,27]$.

The cells grown in the hydrogel nanoscaffolds demonstrate a similar proliferating profile to those of $2 \mathrm{D}$ cultures with a 24-30 h doubling rate. In all three concentrations a spheroid growth pattern was observed, as shown in Figure 5A. After the functionalisation of the matrix with laminin, the growth pattern of ReNcell VM cells was different. Cells hosted in PuraMatrix/laminin 0.5\% scaffolds expressed flat and densely packed cell aggregates (Figure 5B). More loosely composed 3D structures of cells were rarely observed, in those cases mainly after 10 days of cultivation (data not shown). Progenitor cells cultivated in PuraMatrix/laminin 0.25\% showed an increased number of 3D-structures which were already developed after 5 days of cultivation, whereas the number of aggregated forms decreased (Figure 5C). Almost all growth patterns in the 


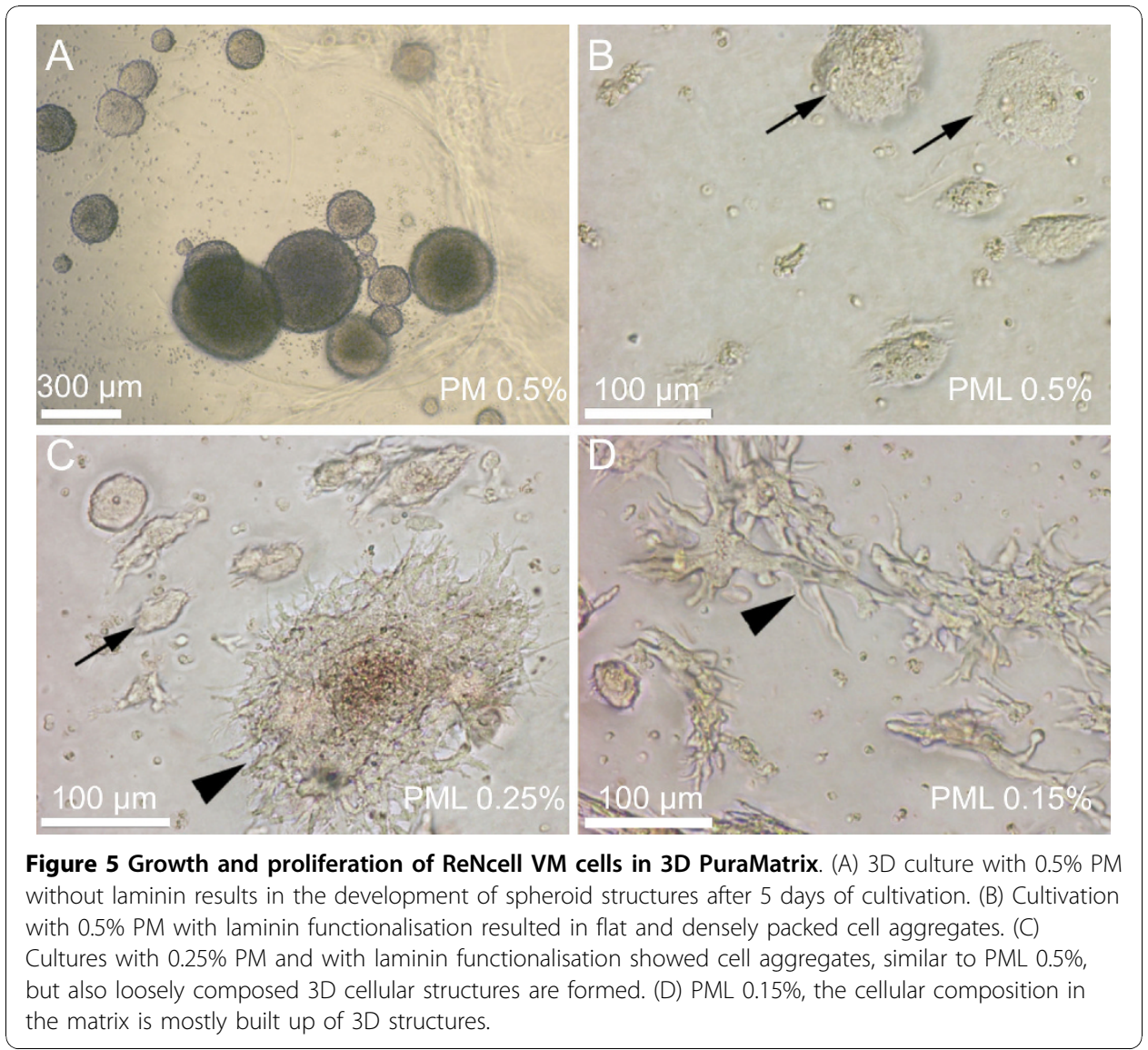

lowest concentration of PuraMatrix/laminin (0.15\%) showed these 3D-structures of ReNcell VM cells. Similar to PuraMatrix/laminin 0.25\% 3D structures were already present after 5 days (Figure 5D). Correspondingly, almost none of the cell aggregates were found within these approaches. These results hint at the conclusion that ReNcell VM cells cultured without surface functionalisation grow in neurospheres, whereas in laminin functionalised matrices the growth pattern of the progenitor cells differs in such a way that high concentrations of the scaffold support the growth of cell aggregates whereas decreasing PuraMatrix concentrations lead to extended 3D-growth of ReNcell VM cells.

\section{Survival of cells in 3D-matrices and 2D-cultivation}

The survival rate of cells cultured in 3D-scaffolds with different PuraMatrix concentrations was analysed by using a Live/Dead assay, performed at days $0,1,4$, and 7 . To determine if there was a benefit of the $3 \mathrm{D}$-matrix over a $2 \mathrm{D}$-culture system we conducted in parallel a live/dead assay in 2D-cultures. An example picture of a live/ dead-assay conducted with cells differentiated for 4 days in a 2D-culture is shown in Figure 6A. Cells differentiated in the 2D-culture system showed a significant increase of dead cells over the time in comparison to day 0 differentiation (Figure 6B, p < 0.005). In comparison no increase of dead cells was observed in the different 3D-scaffolds. Regardless of the PuraMatrix concentration the number of dead cells was stable over the time of differentiation (Figure $6 \mathrm{~B}$ ). The results at day 0 demonstrated in all 

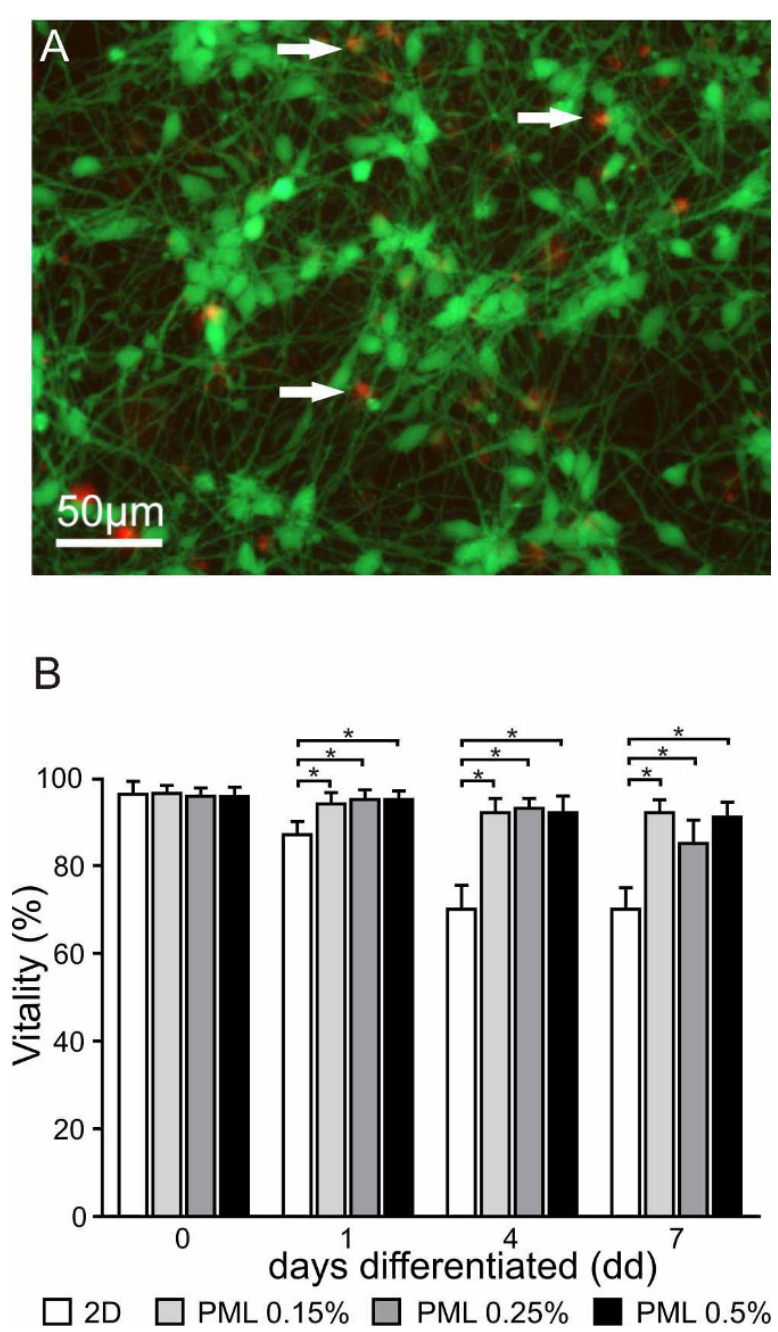

Figure 6 Live/Dead Assay. (A) Live/Dead fluorescence image of ReNcell VM cells in 2D-culture conditions. Green fluorescent cells are alive, red fluorescent nuclei indicate dead cells (arrows). (B) Quantification of the stainings by manual counting of living and dead cells of $2 \mathrm{D}$ and $3 \mathrm{D}$ cultures with different concentrations of PML matrices. Notice the increase of dead cells during differentiation which is significantly higher in the $2 \mathrm{D}$ compared to $3 \mathrm{D}$ cultures. ${ }^{*}$ indicates $p \leq 0.05$.

samples a survival rate higher than $95 \%$. At day 1 the percentage of cell survival in 2Dcultures was about $88 \%$ and it decreased further until about $70 \%$ at day 7 . The lowest survival rate within the three different matrix concentrations was about 85\% (PML $0.25 \%, \mathrm{~d} 7$ ) and therefore only $10 \%$ lower compared to the starting point at day 0 , although this rate was not significantly different from day 0 . From day 1 up to day 7 the survival rates in the $3 \mathrm{D}$-matrices were always significantly higher than in the $2 \mathrm{D}$ cultures ( $p \leq 0.005$ ), indicating that 3D-matrices support the survival of ReNcell VM cells during differentiation.

\section{Neuronal differentiation in 3D-matrices and 2D-cultivation}

Antibody stainings against $\beta$-III-tubulin ( $\beta$ III-tub) and tyrosine hydroxylase (TH) were performed to investigate the influence of the $3 \mathrm{D}$-composition on the number of neurons compared to $2 \mathrm{D}$. The percentage of neuronal cells was determined at day 0 , and 


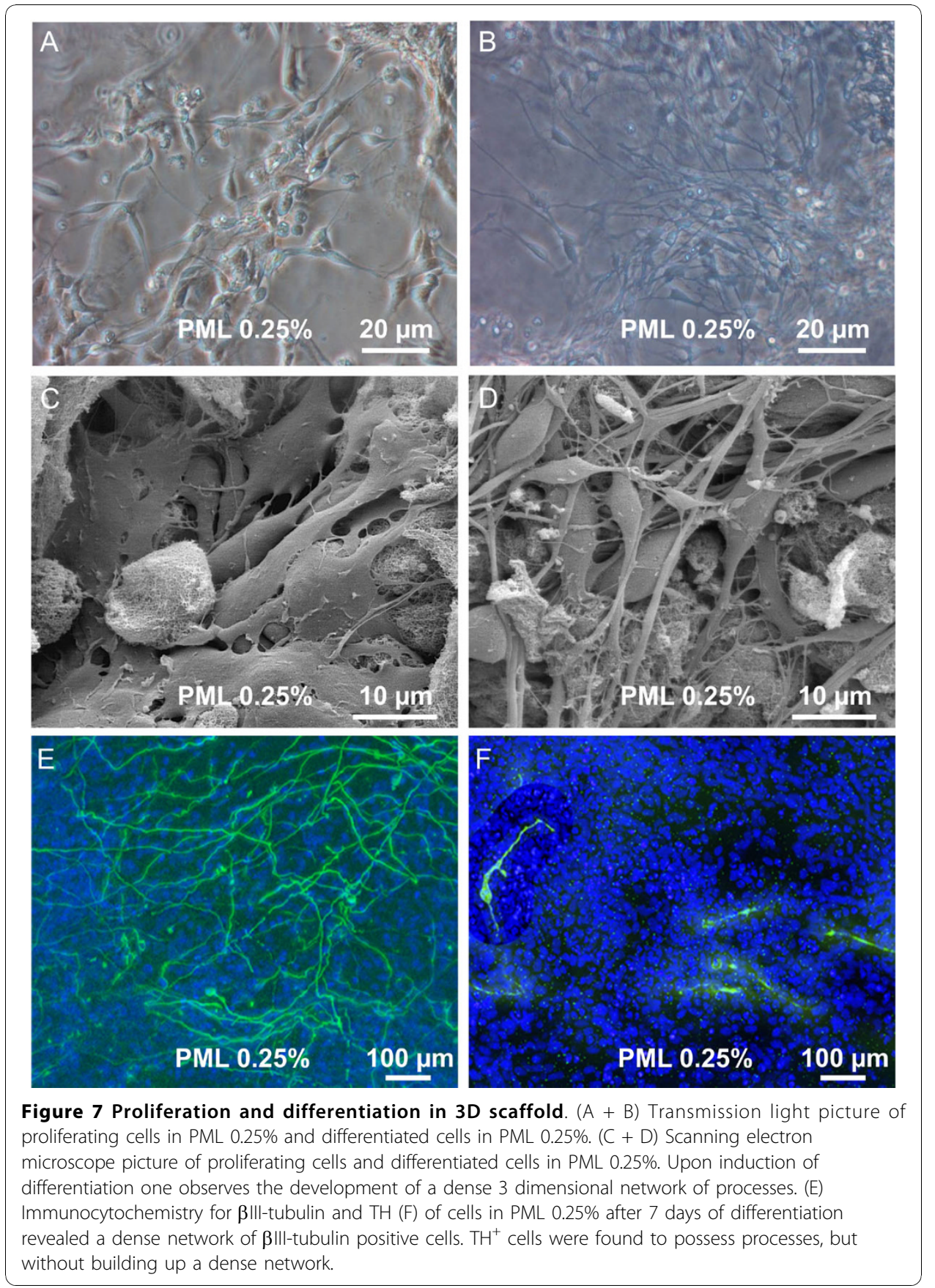

at day 1,4 and 7 after differentiation. Cells were proliferated for up to 4 days in scaffolds consisting of different concentrations of PuraMatrix. Figure 7A shows a transmission light picture of proliferating cells (PM $0.25 \%$ with laminin). Cell bodies with only a few short processes can be seen, a morphology comparable to 2D cultures (data not shown). Investigation of proliferating cells with a scanning electron microscope revealed cell bodies which are embedded in the PuraMatrix (Figure 7C). Upon induction of differentiation, cells started to develop a dense network of processes (Figure 7B and 7D) and began to the express $\beta$ III-tub (Figure 7E) and TH (Figure 7F). 


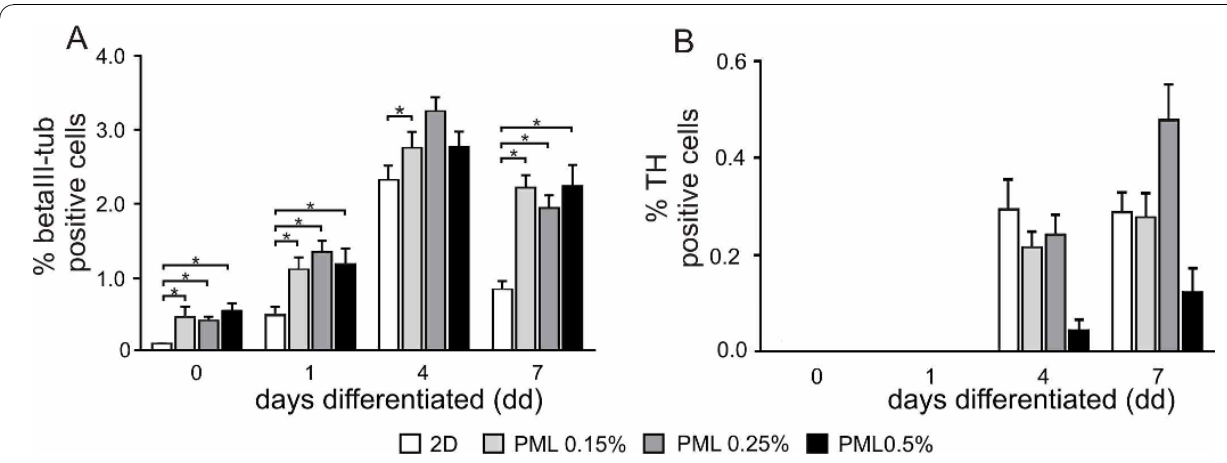

Figure 8 Quantitative analysis of $\beta$ III-tubulin and TH expression. $(A+B)$ Percentage of $\beta$ III-tubulin positive and TH positive cells, respectively, in differentiating ReNcell VM cells grown in 2D and 3D cultures. In all conditions a higher number of $\beta \| l$-tubulin ${ }^{+}$cells was found in the 3D scaffolds in comparison to the $2 \mathrm{D}$ cultures. A PuraMatrix concentration of $0.5 \%$ was found to be adverse for $\mathrm{TH}^{+}$cells as the number was lower in these scaffolds, however the difference was not significant. ${ }^{*}$ indicates $p \leq 0.05$.

In proliferating cells, the percentage of $\beta$ III-tub positive cells $\left(\beta I I I-\right.$ tub $\left.^{+}\right)$ranged between $0.4 \%$ and $0.5 \%$ within the three PuraMatrix/laminin concentrations and was significantly higher in all three PuraMatrix/laminin concentrations compared to the $2 \mathrm{D}$-culture $(0.06 \pm 0.02 \%)$ (Figure $8 \mathrm{~A})$. After one day of differentiation the number of neurons had more than doubled within the PuraMatrix/laminin concentrations, compared to $2 \mathrm{D}$-cultures. At day 7 the number of $\beta$ III-tub ${ }^{+}$cells decreased both within the 3D-scaffolds with all PuraMatrix/laminin concentrations, and in the 2D-cultures. However, in all conditions of the 3D-cultures the number of $\beta$ III-tub ${ }^{+}$cells was still about $2 \%$, higher in comparison to the $2 \mathrm{D}$-cultures $(0.85 \pm 0.09 \%)$.

An example of the detection of $\mathrm{TH}$ positive $\left(\mathrm{TH}^{+}\right)$cells is shown in Figure 7F. At day 0 and 1 of differentiation in none of the conditions $\mathrm{TH}^{+}$cells were found. Expression of $\mathrm{TH}$ was confirmed at day 4 , where $0.2 \%-0.3 \% \mathrm{TH}^{+}$cells were detected within the 2D-culture as well as the two lower concentrations of the PuraMatrix/laminin matrix. In contrast, in the scaffold with the highest PuraMatrix/laminin concentration only $0.04 \pm 0.02 \%$ of $\mathrm{TH}^{+}$cells were found (Figure 8B). At day 7 the scaffolds with a PuraMatrix/laminin concentration of $0.25 \%$ showed the highest number of $\mathrm{TH}^{+}$cells $(0.46 \pm 0.05 \%)$ which was nearly twice the number of $\mathrm{TH}^{+}$cells in the scaffolds with a PuraMatrix/laminin concentration of $0.15 \%(0.27 \pm 0.04)$. Like at day 4 the amount $\mathrm{TH}^{+}$cells was lowest in the scaffolds with a PuraMatrix/laminin concentration of $0.5 \%$ $(0.11 \pm 0.04 \%)$. Regardless of this clear difference the number of $\mathrm{TH}^{+}$cells was not significantly different in any condition. In general, the formation of a 3D-network of $\mathrm{TH}^{+}$ cells can not be confirmed, but $\mathrm{TH}^{+}$cells can be found on different levels of z-stacks which ensures a 3D-growth.

\section{Discussion}

Three-dimensional matrices composed of nanofibres and the use of neural progenitor cells are emerging areas in regenerative medicine. Both applications by themselves promise success as therapeutic tools for neurodegenerative diseases $[1,2,33]$. The combination of both methologies shows synergistic effects and improves the outcome over single applications, as shown in different studies dealing with the culture of cells in 3D scaffolds for example in scaffolds consisting of PuraMatrix [15,22,23]. In our study we investigated the growth and differentiation of a human fetal neural progenitor cell line 
(ReNcell VM, Millipore, USA). These cells can be differentiated into neurons with a dopaminergic phenotype within a short period of cultivation. Although the cells are immortalised and therefore not suitable for clinical application, the fast onset of neuronal differentiation, within $24 \mathrm{~h}$ as shown by the change in morphology and the appearance of markers such as $\beta$-III-tubulin, make them a suitable model system for neuronal differentiation [25-28].

In our study we used the extracellular matrix protein laminin-I which promotes cell adhesion and stimulates neurite outgrowth in various neuronal cell types [34]. Cell adhesion and neurite outgrowth-promoting sites have been identified in the C-terminal site of $\alpha 1$ and $\alpha 2$ chain [35-39], in the cross-region of the molecule [40,41], in the $\gamma 1$ chain $[42,43]$, and in the N-terminal region of the $\alpha 2$ chain [44]. These findings support our primary choice of laminin I as a functionalisation agent in our studies.

In 2D and 3D cultures of ReNcell VM cells there are distinct variations of the growth pattern of functionalised and non-functionalised matrices. Functionalisation with laminin supports cell adhesion and prevents the formation of neurospheres. ReNcell VM cells either attach to the surface of a cell culture flask (2D) or show distinct growth patterns based on the combination of laminin with various PM scaffolds. In this context, the influence of laminin on the matrix structure is of particular interest. Structure and assembly of the functionalised matrix was investigated in detail by AFM microscopy. AFM studies demonstrate that these different growth patterns are linked to the assembly state of the 3D matrix. In general the matrix structure is built from beta-sheets and aggregates or bundles of those. Laminin directly influences the formation of the PM matrices by increasing the number of aggregates (Figure 2 and Figure 3). A PuraMatrix/ laminin concentration of $0.5 \%$ has a dense and closely packed composition so that the distance between fibres is extremely small. Cells cultured in these matrices are not able to build up 3D structures, and grow in a flatter, denser pattern (Figure 5B). Decreasing the PML concentration increases the distance of nanofibres (Figure 3F, D, B) and therefore increases the possibility to form 3D growth patterns (Figure 5C). In addition, we could show that the laminin functionalisation increases the distance between fibres by shifting the composition ratio of beta-sheets to bundles more towards the bundles. Similar influence of laminin on 3D structures was reported for scaffolds consisting of Poly(l-lactic acid), whereas different modifications of the treatment of the scaffolds with laminin resulted in changes of the nanofibre structure and subsequently in the viability and proliferation of PC12 cells [45]. Regarding the changes of the scaffold into the direction of structure with more bundles, it can be concluded that this will result in 3D matrix with higher stiffness and stability. Stiffness of the surrounding matrix has important implications during development, differentiation, disease, and regeneration [46]. Although laminin clearly influences both the scaffold and the growth pattern of the cells in our study, the impact of the stiffness and stability on growth patterns and differentiation was not examined in this study and is a topic for further studies.

Besides the influence of the matrix composition on the growth and proliferation of the cells, we were interested in the question of if a $3 \mathrm{D}$ environment is superior to a $2 \mathrm{D}$ environment, as it is thought to resemble the in vivo situation more closely. Compared to $2 \mathrm{D}$ cultures the total number of cells in all matrix concentrations is significantly higher up to 7 days of differentiation, which supports the importance of a 3D environment. A question which was not addressed in the present study is the role of the non- 
neuronal cells. A high proportion of the cells remain positive for GFAP, suggesting differentiation into glial cells. However, this was observed in the 2D as well as the $3 \mathrm{D}$ culture system (data not shown).

Within this time period the survival rate of cells in the 2D culture system decreased to $70 \%$. In contrast to our findings, Silva et al. [33] could not demonstrate significant differences in cell survival compared to 2D culture within the differentiation phase with the IKVAV hydrogel system. Hence, they suggest that diffusion of nutrients, bioactive factors, and oxygen through these highly hydrated networks is sufficient for survival of large numbers of cells for extended periods of time. The increase of cell survival in 3D matrices was also described by Mahoney and Anseth [47] with poly ethyleneglycol hydrogels. The increase in cell survival during differentiation fits very well with the higher number of $\beta$-III-tubulin positive cells, in particular after 7 days of differentiation (Figure 8). Between day 4 and day 7 of differentiation there is a drop in the number of neurons that is found in both 2D and 3D culture systems. Nevertheless, the total number of neurons remains significantly higher in 3D cultures. Another difference in the growth and differentiation potential of ReNcell VM cells is the increased number of $\beta$-III-tubulin positive neurons at day 0 . Here it might be hypothesised that the functionalised matrix itself has a slightly inductive capacity towards neuronal differentiation. In addition, the growth of cells in a densely and packed environment of the matrix may induce spontaneous differentiation of progenitor cells. Furthermore, our results clearly demonstrate that the PuraMatrix/laminin 0.5\% concentration failed to support the development of dopaminergic neurons at day 4 and day 7 after differentiation. In general, the number of TH-positive cells is reduced compared to 2D cultivation, except for PuraMatrix/laminin $0.25 \%$, in which the number of TH-positive cells was higher after 7 days compared to 2D cultures. This suggests that lower concentrations support differentiation and increase the overall number of differentiated TH-positive cells. This may be due to a correlation between the structure of the matrix, namely the pores and fibres. Thonhoff et al. [23] showed that a concentration of $0.25 \%$ PuraMatrix is optimal to support stem cell differentiation, however these were not functionalised with laminin.

Proceeding from this, further studies may generate new protocols for the enrichment of neurons through manipulating the structure and composition of the matrix. Donato et al. [25] demonstrated that by pre-aggreation of the ReNcell VM cells as neurospheres before differentiation a higher number of neurons could be obtained. Another approach might be to alter the composition of the matrix with defined signalling molecules that promote neuronal differentiation. Silva et al. [33] for instance were able to demonstrate that integration of the laminin epitope IKVAV into a 3D matrix was able to initiate neuronal differentiation. Other authors [21] have demonstrated a wide variety of signalling peptides derived from laminin which have the potential to influence neurite outgrowth and differentiation. Taken together, these results show that besides the known benefit of culturing cells in 3D structures, the composition of the scaffold, specifically the concentration of the matrix material, and the functionalisation of these materials support and enhance growth and differentiation of human neural progenitor cells.

\section{Conclusions}

In this study we demonstrated that dependent on the concentration of PuraMatrix or the functionalisation of PuraMatrix with laminin the overall structure of the 3D 
scaffold is changed. AFM measurements revealed changes in the 3D-structure with respect to the formation of bundles. By analysing the proliferation, differentiation and survival of human neural progenitor cells we showed that the formation and functionalisation of the 3D-structure is important for the fate of the cells. A comparison with other studies dealing with the cultivation of progenitor cells in 3D scaffolds suggests different matrix parameters may best suit different cell lines. Therefore care has to be taken regarding the matrix composition, concentration and functionalisation when using 3D systems. Taking this into account, model systems like the one we describe might be a valuable tool for the generation of defined cell types or might serve as in vitro assays for testing compounds which interact with the proliferation or differentiation of stem and progenitor cells.

\title{
Abbreviations
}

PM: PuraMatrix; PML: PuraMatrix functionalised with laminin

\begin{abstract}
Acknowledgements
We thank Dr. Sergei A. Kuznetsov for the possibility to use the confocal microscope of the Live Cell Imaging Center Rostock. We thank Peter Morgan for the valuable support in the discussion of the data. Finally we thank the European Social Fund (grant number UG 10 022) and the Alfried Krupp Graduate School "Studies of the interaction of free oxygen radicals with molecules at electrodes and applications to biochemical and medical systems" for financial support.
\end{abstract}

\section{Author details}

${ }^{1}$ Albrecht-Kossel-Institute for Neuroregeneration, University of Rostock, Gehlsheimerstrasse 20, 18147 Rostock, Germany. ${ }^{2}$ Institute for Physics, Ernst-Moritz-Arndt University Greifswald, Felix-Hausdorff-Str. 6, 17489 Greifswald, Germany. ${ }^{3}$ Electron Microscopic Centre, Institute of Pathology, University of Rostock, Strempelstrasse 14, 18055 Rostock, Germany. ${ }^{4}$ Division of Cell Biology and Biosystems Technology, Institute of Biological Sciences, University of Rostock, Albert-Einstein-Strasse 3, 18051 Rostock, Germany.

\section{Authors' contributions}

All authors contributed to the drafting and approval of the manuscript. SO: conception/design/analysis of immunocytochemistry data. JS: conception/design and acquisition/analysis immunocytochemistry data. SB: conception/design and acquisition/analysis of AFM data. AL: acquisition/analysis of immunocytochemistry data. LJ: conception/design and acquisition/analysis of SEM data. CAH: conception/design of AFM data.

\section{Competing interests}

The authors declare that they have no competing interests.

Received: 26 November 2009 Accepted: 11 November 2010 Published: 11 November 2010

\section{References}

1. Goldman SA: Neurology and the stem cell debate. Neurology 2005, 64:1675-1676.

2. Martino G, Pluchino S: The therapeutic potential of neural stem cells. Nat Rev Neurosci 2006, 7:395-406

3. Mooney DJ, Baldwin DF, Suh NP, Vacanti JP, Langer R: Novel approach to fabricate porous sponges of poly(D,Llactic-co-glycolic acid) without the use of organic solvents. Biomaterials 1996, 17:1417-1422.

4. Mikos AG, Lyman MD, Freed LE, Langer R: Wetting of poly(L-lactic acid) and poly(DL-lactic-co-glycolic acid) foams for tissue culture. Biomaterials 1994, 15:55-58.

5. Lavik E, Teng YD, Snyder E, Langer R: Seeding neural stem cells on scaffolds of PGA, PLA, and their copolymers. Methods Mol Biol 2002, 198:89-97.

6. Hsu WC, Spilker MH, Yannas IV, Rubin PA: Inhibition of conjunctival scarring and contraction by a porous collagenglycosaminoglycan implant. Invest Ophthalmol Vis Sci 2000, 41:2404-2411.

7. Chamberlain L, Yannas IV, Hsu HP, Strichartz GR, Spector M: Near-terminus axonal structure and function following rat sciatic nerve regeneration through a collagen-GAG matrix in a ten-millimeter gap. J Neurosci Res 2000, 60:666-677.

8. Butler CE, Yannas IV, Compton CC, Correia CA, Orgill DP: Comparison of cultured and uncultured keratinocytes seeded into a collagen-GAG matrix for skin replacements. Br J Plast Surg 1999, 52:127-132.

9. Orgill DP, Butler C, Regan JF, Barlow MS, Yannas IV, Compton CC: Vascularized collagen-glycosaminoglycan matrix provides a dermal substrate and improves take of cultured epithelial autografts. Plast Reconstr Surg 1998, 102:423-429.

10. Chang SC, Rowley JA, Tobias G, Genes NG, Roy AK, Mooney DJ, Vacanti CA, Bonassar LJ: Injection molding of chondrocyte/alginate constructs in the shape of facial implants. J Biomed Mater Res 2001, 55:503-511.

11. Atala A, Cima LG, Kim W, Paige KT, Vacanti JP, Retik AB, Vacanti CA: Injectable alginate seeded with chondrocytes as a potential treatment for vesicoureteral reflux. J Urol 1993, 150:745-747. 
12. Smith IO, Liu XH, Smith LA, Ma PX: Nanostructured polymer scaffolds for tissue engineering and regenerative medicine. Wiley Interdiscip Rev Nanomed Nanobiotechnol 2009, 1:226-236.

13. Hauser CA, Zhang S: Designer self-assembling peptide nanofiber biological materials. Chem Soc Rev 2010, 39:2780-2790.

14. Uemura M, Refaat MM, Shinoyama M, Hayashi H, Hashimoto N, Takahashi J: Matrigel supports survival and neuronal differentiation of grafted embryonic stem cell-derived neural precursor cells. J Neurosci Res 2010, 88:542-551.

15. Holmes TC, de Lacalle S, Su X, Liu G, Rich A, Zhang S: Extensive neurite outgrowth and active synapse formation on self-assembling peptide scaffolds. Proc Natl Acad Sci USA 2000, 97:6728-6733.

16. Leon EJ, Verma N, Zhang S, Lauffenburger DA, Kamm RD: Mechanical properties of a self-assembling oligopeptide matrix. J Biomater Sci Polym Ed 1998, 9:297-312.

17. Zhang S, Holmes T, Lockshin C, Rich A: Spontaneous assembly of a self-complementary oligopeptide to form a stable macroscopic membrane. Proc Natl Acad Sci USA 1993, 90:3334-3338.

18. Nakahara H, Misawa H, Hayashi T, Tanaka M, Yoshida A, Tanaka N, Ozaki T, Kobayashi N: Bone repair using a hybrid scaffold of self-assembling peptide PuraMatrix and polyetheretherketone cage in rats. Cell Transplant 2010, 19:791-797.

19. Gelain F, Unsworth LD, Zhang S: Slow and sustained release of active cytokines from self-assembling peptide scaffolds. J Control Release 2010, 145:231-239.

20. Taraballi F, Natalello A, Campione M, Villa O, Doglia SM, Paleari A, Gelain F: Glycine-spacers influence functional motifs exposure and self-assembling propensity of functionalized substrates tailored for neural stem cell cultures. Front Neuroengineering 2010, 3:1.

21. Semino CE, Merok JR, Crane GG, Panagiotakos G, Zhang S: Functional differentiation of hepatocyte-like spheroid structures from putative liver progenitor cells in three-dimensional peptide scaffolds. Differentiation 2003, 71:262-270.

22. Semino CE, Kasahara J, Hayashi Y, Zhang S: Entrapment of migrating hippocampal neural cells in three-dimensional peptide nanofiber scaffold. Tissue Eng 2004, 10:643-655.

23. Thonhoff JR, Lou DI, Jordan PM, Zhao X, Wu P: Compatibility of human fetal neural stem cells with hydrogel biomaterials in vitro. Brain Res 2008, 1187:42-51.

24. Erickson IE, Huang AH, Chung C, Li RT, Burdick JA, Mauck RL: Differential maturation and structure-function relationships in mesenchymal stem cell- and chondrocyte-seeded hydrogels. Tissue Eng Part A 2009, 15:1041-1052

25. Donato R, Miljan EA, Hines SJ, Aouabdi S, Pollock K, Patel S, Edwards FA, Sinden JD: Differential development of neuronal physiological responsiveness in two human neural stem cell lines. BMC Neurosci 2007, 8:36.

26. Morgan PJ, Ortinau S, Frahm J, Kruger N, Rolfs A, Frech MJ: Protection of neurons derived from human neural progenitor cells by veratridine. Neuroreport 2009, 20:1225-1229.

27. Schmöle AC, Brennführer A, Karapetyan G, Jaster R, Pews-Davtyan A, Ortinau S, Beller M, Rolfs A, Frech M: Novel indolylmaleimide acts as GSK-3 $\beta$ inhibitor in human neural progenitor cells. Bioorg Med Chem 2010, 18:6785-6795.

28. Hübner R, Schmöle AC, Liedmann A, Frech MJ, Rolfs A, Luo J: Differentiation of human neural progenitor cells regulated by Wnt-3a. Biochem Biophys Res Commun 2010, 40:358-362.

29. Pews-Davtyan A, Tillack A, Schmöle AC, Ortinau S, Frech MJ, Rolfs A, Beller M: A new facile synthesis of 3-amidoindole derivatives and their evaluation as potential GSK-3beta inhibitors. Org Biomol Chem 2010, 8:1149-1153.

30. Yokoi H, Kinoshita T, Zhang S: Dynamic reassembly of peptide RADA16 nanofiber scaffold. Proc Natl Acad Sci USA 2005, 102:8414-8419.

31. Turkevich J, Stevenson P, Hillier J: A study of the nucleation and growth processes in the synthesis of colloidal gold. Discuss Faraday Soc 1951, 11:55-75.

32. Zhang S, Holmes TC, DiPersio CM, Hynes RO, Su X, Rich A: Self-complementary oligopeptide matrices support mammalian cell attachment. Biomaterials 1995, 16:1385-1393.

33. Silva GA: Neuroscience nanotechnology: progress, opportunities and challenges. Nat Rev Neurosci 2006, 7:65-74.

34. Powell SK, Rao J, Roque E, Nomizu M, Kuratomi Y, Yamada Y, Kleinman HK: Neural cell response to multiple novel sites on laminin-1. J Neurosci Res 2000, 61:302-312.

35. Tashiro K, Sephel GC, Weeks B, Sasaki M, Martin GR, Kleinman HK, Yamada Y: A synthetic peptide containing the IKVAV sequence from the A chain of laminin mediates cell attachment, migration, and neurite outgrowth. $J$ Biol Chem 1989, 264:16174-16182.

36. Skubitz AP, Letourneau PC, Wayner E, Furcht LT: Synthetic peptides from the carboxy-terminal globular domain of the A chain of laminin: their ability to promote cell adhesion and neurite outgrowth, and interact with heparin and the beta 1 integrin subunit. J Cell Biol 1991, 115:1137-1148.

37. Calof AL, Campanero MR, O'Rear JJ, Yurchenco PD, Lander AD: Domain-specific activation of neuronal migration and neurite outgrowth-promoting activities of laminin. Neuron 1994, 13:117-130.

38. Richard BL, Nomizu M, Yamada Y, Kleinman HK: Identification of synthetic peptides derived from laminin alpha1 and alpha2 chains with cell type specificity for neurite outgrowth. Exp Cell Res 1996, 228:98-105.

39. Nomizu M, Kim WH, Yamamura K, Utani A, Song SY, Otaka A, Roller PP, Kleinman HK, Yamada Y: Identification of cell binding sites in the laminin alpha 1 chain carboxyl-terminal globular domain by systematic screening of synthetic peptides. J Biol Chem 1995, 270:20583-20590.

40. Edgar D, Timpl R, Thoenen $\mathrm{H}$ : The heparin-binding domain of laminin is responsible for its effects on neurite outgrowth and neuronal survival. EMBO J 1984, 3:1463-1468.

41. Tashiro K, Nagata I, Yamashita N, Okazaki K, Ogomori K, Tashiro N, Anai M: A synthetic peptide deduced from the sequence in the cross-region of laminin A chain mediates neurite outgrowth, cell attachment and heparin binding. Biochem J 1994, 302(Pt 1):73-79.

42. Liesi P, Narvanen A, Soos J, Sariola H, Snounou G: Identification of a neurite outgrowth-promoting domain of laminin using synthetic peptides. FEBS Lett 1989, 244:141-148.

43. Nomizu M, Kuratomi Y, Song SY, Ponce ML, Hoffman MP, Powell SK, Miyoshi K, Otaka A, Kleinman HK, Yamada Y: Identification of cell binding sequences in mouse laminin gamma1 chain by systematic peptide screening. J Biol Chem 1997, 272:32198-32205. 
44. Nomizu M, Song SY, Kuratomi Y, Tanaka M, Kim WH, Kleinman HK, Yamada Y: Active peptides from the carboxylterminal globular domain of laminin alpha2 and Drosophila alpha chains. FEBS Lett 1996, 396:37-42.

45. Koh HS, Yong T, Chan CK, Ramakrishna S: Enhancement of neurite outgrowth using nano-structured scaffolds coupled with laminin. Biomaterials 2008, 29:3574-3582.

46. Discher DE, Janmey P, Wang YL: Tissue cells feel and respond to the stiffness of their substrate. Science 2005, 310:1139-1143.

47. Mahoney MJ, Anseth KS: Three-dimensional growth and function of neural tissue in degradable polyethylene glycol hydrogels. Biomaterials 2006, 27:2265-2274.

doi:10.1186/1475-925X-9-70

Cite this article as: Ortinau et al: Effect of 3D-scaffold formation on differentiation and survival in human neural progenitor cells. BioMedical Engineering OnLine 2010 9:70.

Submit your next manuscript to BioMed Central and take full advantage of:

- Convenient online submission

- Thorough peer review

- No space constraints or color figure charges

- Immediate publication on acceptance

- Inclusion in PubMed, CAS, Scopus and Google Scholar

- Research which is freely available for redistribution 